Queensland, Royal Brisbane and Women's Hospital, Herston, Qld, Australia

Background: Improvements in fungal species identification in the clinical laboratory has seen an increased recognition of cryptic Aspergillus species as a cause of invasive aspergillosis. Case: An 18-year-old male presented to hospital after a 1-month history of nasal congestion and facial headache. He had no significant medical history. An initial CT sinuses showed evidence of chronic sinusitis. He was discharged with oral amoxicillinclavulanate and prednisolone with a plan for outpatient management. Shortly thereafter he developed CNIII and CNIV palsy and was admitted to hospital with an MRI brain showing an expansile mass centred on the clivus extending into the posterior and pituitary fossa. A surgical procedure and sinus tissue biopsy was taken which demonstrated fungal hyphae. IV liposomal amphotericin B and posaconazole were commenced. Suede-like beige colonies were noted on the Sabouraud agar plate from culture of intraoperative specimens. Internal transcribed spacer (ITS) region sequencing (577 bp) identified it as Aspergillus felis. Despite antifungal therapy, his CNS infection progressed, and he died 2 weeks from admission to hospital.

Conclusion: Aspergillus felis is a member of the A. viridinutans complex. It was named after the first host in which clinical disease was described. This is the first documented death in a human from A. felis infection.

\section{THELAZIASIS AS AN UNCOMMON CAUSE OF GIANT PAPILLARY CONJUNCTIVITIS}

Sandy Pui-shan Cheung ${ }^{1}$, Yat-ming Tsang ${ }^{2}$, Kristine Shik Luk ${ }^{2}$, Alex Yat-man $\mathrm{Ho}^{2}$, Leo Wai-lok Chan ${ }^{1}$, Robert Fung Lam ${ }^{1}$, Wing-kin To ${ }^{2}$

${ }^{1}$ Department of Ophthalmology, Caritas Medical Centre, Hong Kong, People's Republic of China; ${ }^{2}$ Department of Pathology, Princess Margaret Hospital, Hong Kong, People's Republic of China

We report a local human case of Thelazia callipaeda eye infection in a 49-year-old lady who presented with right eye foreign body sensation for 1 month. She recalled a fly being stuck onto her right upper eyelashes with mascara when she went hiking in a forest trail in Hong Kong. On assessment there were a lot of giant papillae on palpebral conjunctiva. Three living worms crawling on the surface of conjunctiva were removed by the ophthalmologist. The worm was identified as female Thelazia callipaeda based on the anterior location of vaginal opening relative to the oesophago-intestinal junction and the wide cuplike buccal capsule. ${ }^{1}$ Identification was confirmed with molecular sequencing of the D1 and D2 domains of $28 \mathrm{~s}$ rDNA. ${ }^{2}$ After removal, her symptoms resolved completely. Human thelaziasis is probably under-reported in many countries including Hong Kong. Stray dogs or monkeys probably serve as the reservoir hosts in Hong Kong. The presence of giant papillary conjunctivitis in non-contact lens wearers should alert clinicians to the possibility of thelaziasis in patients with compatible exposure history in endemic regions. Molecular confirmation of identity is particularly helpful when the morphological differences between species cannot be well appreciated.

\section{References}

1. Shen J, Gasser RB, Chu D, et al. Human thelaziosis-a neglected parasitic disease of the eye. J Parasitol 2006; 92: $872-6$.

2. Gouÿ J, Ferté H, Depaquit J, et al. Phylogeny of the Trichostrongylina (Nematoda) inferred from 28S rDNA sequences. Mol Phylogenet Evol 2001; 19: 430-42.

\section{IDENTIFYING ORGANISMS DIRECTLY FROM BACT/ ALERT FAN PLUS BLOOD CULTURE BOTTLES USING THE BIOMÉRIEUX MALDI-TOF MS SYSTEM IN A LARGE LABORATORY NETWORK}

\author{
Harry N. Walker ${ }^{1,2}$ \\ ${ }^{1}$ Dorevitch Pathology, Vic, Australia; ${ }^{2}$ Department of Infectious \\ Diseases, Western Health, Vic, Australia
}

Identifying organisms directly from positive blood culture bottles using MALDI-TOF MS has many advantages to patients, clinical services, and laboratories. However, few published methods have used both BacT/Alert culture media and Vitek MS (both BioMerieux, France). The effect of transporting bottles on test performance has also not been assessed. In this study, 802 positive blood culture bottles were analysed, including 234 requiring inter-laboratory transport, using a method described recently. ${ }^{1}$ Correct identification rates were high for $S$. aureus (100\% of new diagnostic samples), E. faecalis (100\%), Gram negative bacilli (90.9\%), and coagulase-negative Staphylococcus species $(81.8 \%)$. Three false identifications were made, none with clinical significance. For Gram positive cocci in pairs or chains, more correct identifications were made from bottles analysed immediately compared to transported bottles (OR 2.65, $p=0.016$ ), and longer transport time was associated with lower probability of correct identification (OR 0.984 per additional hour, $p=0.040$ ). The first step in the protocol was then omitted, and a validation with 170 paired samples showed no significant differences. This technique is a vastly more cost-effective alternative to molecular techniques, and is valid for use on bottles after transport times of many hours.

\section{Reference}

1. Samaranayake W, Dempsey S, Howard-Jones AR, et al. Rapid direct identification of positive paediatric blood cultures by MALDI-TOF MS technology and its clinical impact in the paediatric hospital setting. BMC Res Notes 2020; 13: 12.

\section{WHOLE GENOME SEQUENCING FOR THE $M$. TUBERCULOSIS RESISTOME}

$\underline{\text { Ivan Bastian }}$

MID Directorate, SA Pathology, Adelaide, SA, Australia

The SARS-CoV-2 pandemic has trail-blazed the coordinated WGS of an infectious agent in each jurisdiction with AusTrakka providing a shared national analysis pipeline and database. The Communicable Diseases Genomics Network (CDGN) and the National Tuberculosis Advisory Committee (NTAC) have now identified Mycobcterium tuberculosis as a priority organism for WGS rollout in 2021/2. 
A CDGN sub-committee with NTAC and other representatives has: surveyed TB WGS in the jurisdictions; compared methodologies; and optimised formats for reporting TB WGS results to clinicians and the state TB Units. Different methodologies and analysis pipelines are used in the jurisdictions. An external quality assurance program is being explored with RCPAQAP to ensure comparable resistome results are obtained. To assist with this TB WGS standardisation, WHO has matched genomic and phenotypic susceptibility results for a range of drugs for thousands of isolates to produce a valuable database for integration into TB resistome analyses. The CDGN sub-committee is also investigating performing cross-jurisdictional cluster investigations using AusTrakka and wgMLST.

This presentation will highlight the opportunities and the challenges facing TB WGS. Susceptibility testing of non-tuberculous mycobacteria (NTM), another challenging field, will also be mentioned.

\section{UPDATE ON MYCOBACTERIAL VACCINES}

Angelo A. Izzo

Tuberculosis Research Group, Centenary Institute/University of Sydney, Royal Prince Alfred Hospital, Sydney, NSW, Australia

As we celebrate 100 years since the first use of the bacillus Calmette-Guérin (BCG) vaccine, the only licensed vaccine against tuberculosis, this is an opportune time to examine the current state of tuberculosis vaccine development. With a better understanding of the host innate and adaptive immune response to infection and how Mycobacterium tuberculosis grows and subverts this response, vaccines are being made to accommodate use in different populations. There are a diverse range of tuberculosis vaccine formulations currently in development, with the intent of designing these vaccines to target the specific stages of infection such as prevention of infection/disease or therapeutic use as stand-alone or as an adjunct to drug treatment. With more vaccines reaching clinical trials, we are understanding how to make better vaccines and also determine how BCG can be incorporated into the vaccination regimen. However, many questions remain about the best way to move forward to provide vaccines that can eliminate the deadly pandemic. The presentation will provide an overview of the general strategies for moving vaccines through the development pipeline.

\section{EXPERIENCE WITH A TRIPLEX ARBOVIRUS NUCLEIC ACID TEST (NAT) AT A CANADIAN PUBLIC HEALTH LABORATORY}

Saugata Choudhury $^{1,2,3}$, Raymond Tellier ${ }^{4}$, Kevin Fonseca ${ }^{2,5}$, Byron M. Berenger $^{2,3}$

${ }^{1}$ Dorevitch Laboratories, Heidelberg, Melbourne, Vic, Australia; ${ }^{2}$ Public Health Laboratory, Alberta Precision Laboratories, Calgary, Alberta, Canada; ${ }^{3}$ Department of Pathology and Laboratory Medicine, Cumming School of Medicine, University of Calgary, Calgary, Alberta, Canada; ${ }^{4}$ Division of Infectious Diseases, Department of Medicine, McGill University, Montreal, Canada; ${ }^{5}$ Department of Microbiology, Immunology and Infectious Diseases, Cumming School of Medicine, University of Calgary, Calgary, Alberta, Canada
Dengue, Chikungunya and Zika infections occur in tropical and subtropical regions. We illustrate the application of an in-house multiplex NAT targeting these arboviruses for returning travellers in Alberta, Canada. NAT was performed until 40 days from symptom onset due to protracted Zika viruria. From 1 September 2017 - 31 August 2019, 2552 specimens from 1932 patients were tested. Approximately $2 \%$ of travellers were NAT positive for dengue $(n=42)$, Chikungunya $(n=4)$, and Zika $(n=1)$. Major symptoms were fever, myalgia and rash. Popular destinations included SouthEast Asia (68.5\%), South America (25\%) and the Caribbean (6.5\%). Ct values were lower ( 1.5 logs) within $1-3$ days post-exposure than for those presenting subsequently. Nineteen patients had urine and plasma submitted; 5 were positive for both specimens and 2 were positive only for dengue in the urine. $\mathrm{Ct}$ values were stronger for plasma as compared to the corresponding urine. Dengue RNA was detectable until 10 days and 5 days post-exposure in plasma and urine respectively. Owing to dengue viraemia detected beyond the conventional 7 days and a global decline in circulating Zika, a cutoff of 14 days from symptom onset to NAT is deemed sufficient. Inclusion of a zoonotic history collection form results in improved test utilisation.

\section{THE ART OF DIAGNOSTIC STEWARDSHIP: URINARY TRACT INFECTION}

Michael Addidle

Pathlab, New Zealand

Diagnostic stewardship is an increasingly important concept within diagnostic microbiology laboratories, leading to potentially increased quality of results, decreased antimicrobial prescribing, and cost efficiencies. Urine samples in particular are a good area to focus diagnostic stewardship efforts, due to high numbers of urines submitted to the laboratory and the significant potential for inappropriate treatment based on the result. Clinical microbiologists have an important leadership role in promoting good diagnostic stewardship policies for processing of urine samples within the microbiology laboratory. This may include diagnostic stewardship interventions at all stages of the testing process; pre-analytical, analytical and post-analytical. A literature review is presented reviewing the latest research in the field of 'urinary' diagnostic stewardship, along with the author's own experiences in implementing various diagnostic stewardship measures for urine samples in a large regional diagnostic microbiology laboratory.

\section{A REVIEW OF CLOSTRIDIUM DIFFICILE TESTING ALGORITHMS FROM THE RCPAQAP EQA PROGRAM}

Katherine Ryan

Royal College of Pathologists of Australasia Quality Assurance Programs (RCPAQAP), NSW, Australia

Background: The Royal College of Pathologists of Australasia Quality Assurance Programs (RCPAQAP) offers external quality assurance (EQA) for pathology laboratories in Australia and over 60 other countries.

Clostridioides (formerly Clostridium) difficile infection (CDI) has emerged as a serious public health threat in many countries. 